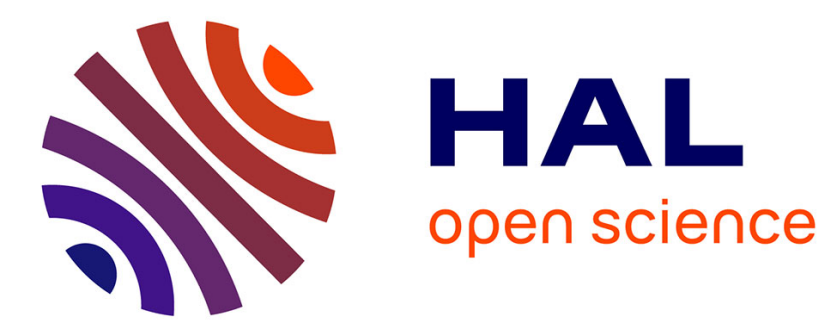

\title{
Thin-plate theory for large elastic deformations
}

David Steigmann

\section{To cite this version:}

David Steigmann. Thin-plate theory for large elastic deformations. International Journal of NonLinear Mechanics, 2007, 42 (2), pp.233-240. hal-00783037

\section{HAL Id: hal-00783037 \\ https://hal.science/hal-00783037}

Submitted on 31 Jan 2013

HAL is a multi-disciplinary open access archive for the deposit and dissemination of scientific research documents, whether they are published or not. The documents may come from teaching and research institutions in France or abroad, or from public or private research centers.
L'archive ouverte pluridisciplinaire HAL, est destinée au dépôt et à la diffusion de documents scientifiques de niveau recherche, publiés ou non, émanant des établissements d'enseignement et de recherche français ou étrangers, des laboratoires publics ou privés. 


\title{
Thin-plate theory for large elastic deformations
}

\author{
David J. Steigmann \\ Department of Mechanical Engineering, 6133 Etcheverry Hall, University of California, Berkeley, CA 94720, USA
}

Dedicated to the memory of R.S. Rivlin

\begin{abstract}
Non-linear plate theory for thin prismatic elastic bodies is obtained by estimating the total three-dimensional strain energy generated in response to a given deformation in terms of the small plate thickness. The Euler equations for the estimate of the energy are regarded as the equilibrium equations for the thin plate. Included among them are algebraic formulae connecting the gradients of the midsurface deformation to the through-thickness derivatives of the three-dimensional deformation. These are solvable provided that the three-dimensional strain energy is strongly elliptic at equilibrium. This framework yields restrictions of the Kirchhoff-Love type that are usually imposed as constraints in alternative formulations. In the present approach they emerge as consequences of the stationarity of the energy without the need for any a priori restrictions on the three-dimensional deformation apart from a certain degree of differentiability in the direction normal to the plate.
\end{abstract}

Keywords: Non-linear elasticity; Plate theory; Strong ellipticity

\section{Introduction}

The derivation of two-dimensional theories of elastic plates from three-dimensional elasticity is one of the main open problems in solid mechanics. This problem has a long and rich history which has seen the development of many ideas about the manner in which the three-dimensional aspects of deformation should best be represented in a two-dimensional model [1]. In recent years the subject has been revisited using modern asymptotic and variational methods. In particular, for equilibrium problems a number of works [2-4] based on the method of Gamma convergence [5] have furnished rigorous foundations for some theories which were earlier proposed on the basis of more formal reasoning. The large body of recent work emphasizing alternative approaches based on asymptotic methods is described in [6,7] and summarized in [8]. It is notable that both approaches yield essentially the same models depending on how boundary conditions are imposed and the manner in which the applied loads scale relative to

E-mail address: steigman@me.berkeley.edu. plate thickness. Another approach, developed in [9], is based on a set of exact two-dimensional necessary conditions for the three-dimensional balance laws augmented by constitutive equations involving the variables appearing in the twodimensional theory. Alternative ideas involve the imposition of a priori constraints on the three-dimensional deformation intended to represent its through-thickness variation in a thin region [10]. In contrast, the direct theories are based a priori on the concept of a deformable surface [11] endowed with vector fields intended to capture certain aspects of the threedimensional deformation of an actual thin body.

A significant lacuna in modern works concerned with the relationship between the two- and three-dimensional theories is the lack of a single model containing a small parameter (the plate thickness) applicable to problems involving combined bending and stretching [4]. In particular, the method of Gamma convergence has thus far failed to furnish a model in which both effects occur simultaneously. Likewise, asymptotic methods are typically based on global asymptotic expansions of the weak forms of the equilibrium equations, leading to model equations without a small parameter that are uniformly valid over the entire plate [6]. In contrast, classical models 
incorporating a small parameter lead to boundary-layer phenomena in which stretching typically dominates over much of the plate while bending dominates locally as needed to accommodate boundary data or abrupt changes in geometry or the distribution of loading. Such models incorporate both modes of deformation in a single framework which is amenable to conventional methods of analysis based on matched asymptotic expansions.

The present work is aimed at furnishing a non-linear model of precisely this kind. The approach used is to estimate the energy of a thin body generated in response to a given kinematically possible three-dimensional deformation. This generates an expansion of the plate energy in powers of thickness which is truncated at a level that incorporates stretching and bending simultaneously. The relevant expression is developed in Section 2. In Section 3 it is shown that this expression subsumes the extremes associated with membrane and inextensional bending behavior, both of which have been the subject of independent analyses by the method of Gamma convergence [2,12]. The tacit assumption is that the terms retained in our expansion dominate those not retained. In general this needs to be confirmed a posteriori after the solution to a specific problem is obtained. The same remark applies to works based on asymptotic expansions or on the method of Gamma convergence. To date neither approach has yielded theorems on the convergence of thickness expansions of the energy. The equilibrium equations of the model are deduced in Section 4 from the stationarity of the truncated energy. Associated boundary conditions are discussed in Section 5.

The idea pursued here is similar in spirit to that developed in [13-15], albeit with some differences. In particular, some of the Euler equations derived here are of an algebraic type. These specify relationships among the deformation of the plate midsurface and certain vector fields representing the through-thickness derivatives of the three-dimensional deformation. Thus, in principle these relations pertain to equilibrium fields. Similar restrictions are obtained in [14,15] and assumed to apply to all deformations of the plate, not merely those that satisfy equilibrium conditions. Consequently our approach is less restrictive, allowing for the possibility that different relationships may exist among these variables in a dynamical setting.

The present development is based on the standard purely mechanical theory of finite elasticity. The associated Piola stress $\mathbf{P}$ is given by

$\mathbf{P}(\tilde{\mathbf{F}})=U_{\tilde{\mathbf{F}}}$,

the gradient with respect to the deformation gradient $\tilde{\mathbf{F}}$ of the strain energy $U(\tilde{\mathbf{F}})$ per unit reference volume. This is assumed for the sake of simplicity to be independent of $\mathbf{x}$, the position in a reference configuration $\kappa$ of a material point of the elastic body. The force per unit area transmitted across a surface in the reference configuration with unit normal $\mathbf{N}$ is $\mathbf{p}(\mathbf{N})=\mathbf{P}(\tilde{\mathbf{F}}) \mathbf{N}$. The deformation gradient satisfies $\mathrm{d} \mathbf{y}=\tilde{\mathbf{F}} \mathrm{d} \mathbf{x}$, where $\mathbf{y}=\chi(\mathbf{x})$ is the position after deformation of the same material point and $\chi$ is the deformation function. We assume throughout that equi- librium deformations satisfy the well-known strong-ellipticity condition

$\mathbf{a} \otimes \mathbf{b} \cdot \mathscr{M}(\tilde{\mathbf{F}})[\mathbf{a} \otimes \mathbf{b}]>0 \quad$ for all $\mathbf{a} \otimes \mathbf{b} \neq \mathbf{0}$,

where

$\mathscr{M}(\tilde{\mathbf{F}})=U_{\tilde{\mathbf{F}} \tilde{\mathbf{F}}}$

is the tensor of elastic moduli. Here and elsewhere we use notation defined, in terms of Cartesian components, by $\mathscr{M}[\mathbf{A}]=$ $\mathscr{M}_{i A j B} A_{j B} \mathbf{e}_{i} \otimes \mathbf{E}_{A}$ for any second-order tensor $\mathbf{A}$, where Latin indices take values in $\{1,2,3\},\left\{\mathbf{e}_{i}\right\}$ and $\left\{\mathbf{E}_{A}\right\}$ are orthonormal bases, and $\mathscr{M}_{i A j B}=\partial^{2} U / \partial F_{i A} \partial F_{j B}$. The scalar product of second-order tensors $\mathbf{A}, \mathbf{B}$ is $\mathbf{A} \cdot \mathbf{B}=\operatorname{trace}\left(\mathbf{A}^{\mathrm{t}} \mathbf{B}\right)=A_{i A} B_{i A}$, where the superscript ${ }^{\mathrm{t}}$ is used to denote the transpose.

It is well known that the restriction (2) on the moduli is necessary for the stability of a homogeneously deformed equilibrium state against infinitesimal plane harmonic waves. We will also have need of the tensor of second-order moduli [16]

$\mathscr{A}(\tilde{\mathbf{F}})=U_{\tilde{\mathbf{F}} \tilde{\mathbf{F}} \tilde{\mathbf{F}}}$

In Section 4 we use this in conjunction with notation defined by $\mathscr{A}[\mathbf{A}][\mathbf{B}]=\mathscr{A}_{i A j B k C} A_{j B} B_{k C} \mathbf{e}_{i} \otimes \mathbf{E}_{A}$, where $\mathscr{A}_{i A j B k C}=$ $\partial^{3} U / \partial F_{i A} \partial F_{j B} \partial F_{k C}$.

In general the strain-energy function is subject to further restrictions associated with frame invariance and material symmetry but these are not germane to the issues of concern to us here. When we stipulate that (2) applies to equilibrium deformations, we intend that it apply to all deformations that satisfy the Euler equations and relevant boundary conditions generated by the particular thin-plate potential energy functional considered. Thus we do not impose strong ellipticity as a constitutive hypothesis for arbitrary deformations, nor do we adopt any other constitutive hypothesis. In this respect our approach is less restrictive than others based, for example, on the method of Gamma convergence.

A plate is a material body identified with a reference configuration $\kappa$ generated by the parallel translation of a simply connected plane region $\Omega$, with piecewise smooth boundary curve $\partial \Omega$, in the direction orthogonal to $\Omega$. The body itself occupies the volume $\bar{\Omega} \times(-h / 2, h / 2)$, where $\bar{\Omega}=\Omega \cup \partial \Omega$ and $h$ is the (uniform) thickness. Let $l$ be another length scale such as the diameter of $\Omega$. We assume that $\varepsilon:=h / l \ll 1$. Further, we regard $l$ as a fixed scale and adopt it as the measure of length. This allows us to set $l=1$ and thus to simplify the notation. The discussion is further simplified by taking the material of the body to have uniform properties.

\section{Small-thickness estimate of the energy}

The reference placement of the plate is described by the three-dimensional normal-coordinate parametrization

$\mathbf{x}=\mathbf{u}+\varsigma \mathbf{k}$,

where $\mathbf{u} \in \Omega$ and $\varsigma \in(-\varepsilon / 2, \varepsilon / 2)$. We assume the origin of the position $\mathbf{x}$ to lie on $\Omega$. The projection

$\mathbf{1}=\mathbf{I}-\mathbf{k} \otimes \mathbf{k}$, 
where $\mathbf{I}$ is the identity for $E^{3}$, is the (two-dimensional) identity on the translation (vector) space $\Omega^{\prime}$ of $\Omega$, and may be used to expand $\tilde{\mathbf{F}}=\tilde{\mathbf{F}} \mathbf{I}$ in the form

$\tilde{\mathbf{F}}=\mathbf{F}+\mathbf{d} \otimes \mathbf{k}$

where

$\mathbf{F}=\tilde{\mathbf{F}} \mathbf{1}$ and $\mathbf{d}=\tilde{\mathbf{F}} \mathbf{k}$.

Using $\mathrm{d} \mathbf{y}=\tilde{\mathbf{F}} \mathrm{d} \mathbf{x}$ with $\hat{\mathbf{y}}(\mathbf{u}, \varsigma)=\chi(\mathbf{u}+\varsigma \mathbf{k})$ and $\mathrm{d} \mathbf{u} \in \Omega^{\prime}$ yields the alternative representation

$(\tilde{\mathbf{F}} \mathbf{1}) \mathrm{d} \mathbf{u}+\tilde{\mathbf{F}} \mathbf{k} \mathrm{d} \varsigma=\mathrm{d} \hat{\mathbf{y}}=(\nabla \hat{\mathbf{y}}) \mathrm{d} \mathbf{u}+\hat{\mathbf{y}}^{\prime} \mathrm{d} \varsigma$,

where $\nabla()$ is the (two-dimensional) gradient with respect to $\mathbf{u}$ at fixed $\varsigma$ and the notation ()$^{\prime}$ is used to denote $\partial() / \partial \varsigma$ at fixed u. It follows that

$\mathbf{F}=\nabla \hat{\mathbf{y}} \quad$ and $\quad \mathbf{d}=\hat{\mathbf{y}}^{\prime}$

The total strain energy $\mathscr{E}$ in a given deformation is

$\mathscr{E}=\int_{\kappa} U(\tilde{\mathbf{F}}(\mathbf{x})) \mathrm{d} V=\int_{\Omega} \int_{-\varepsilon / 2}^{\varepsilon / 2} U(\hat{\mathbf{F}}(\mathbf{u}, \varsigma)) \mathrm{d} \varsigma \mathrm{d} A$,

where $\hat{\mathbf{F}}(\mathbf{u}, \varsigma)=\tilde{\mathbf{F}}(\mathbf{u}+\varsigma \mathbf{k})$. We write the through-thickness integral in the form

$I(\varepsilon)=\int_{-\varepsilon / 2}^{\varepsilon / 2} G(\varsigma) \mathrm{d} \varsigma$,

where $G(\cdot)=U(\hat{\mathbf{F}}(\mathbf{u}, \cdot))$. If $\tilde{\mathbf{F}}(\mathbf{x})$ is a $C^{4}$ function (so that $\chi(\mathbf{x})$ is $C^{5}$ ), then by Leibniz' rule and Taylor's theorem,

$I(\varepsilon)=\varepsilon G_{0}+\frac{1}{24} \varepsilon^{3} G_{0}^{\prime \prime}+\frac{1}{16(5 !)} \varepsilon^{5} G^{\prime \prime \prime \prime}(\bar{\varsigma})$

for some $\bar{\varsigma} \in(-\varepsilon / 2, \varepsilon / 2)$, where the subscript ()$_{0}$ identifies function values at $\varsigma=0$ and where, by the chain rule,

$G_{0}=U\left(\hat{\mathbf{F}}_{0}\right), \quad G_{0}^{\prime}=\mathbf{P}\left(\hat{\mathbf{F}}_{0}\right) \cdot \hat{\mathbf{F}}_{0}^{\prime} \quad$ and

$G_{0}^{\prime \prime}=\mathscr{M}\left(\hat{\mathbf{F}}_{0}\right)\left[\hat{\mathbf{F}}_{0}^{\prime}\right] \cdot \hat{\mathbf{F}}_{0}^{\prime}+\mathbf{P}\left(\hat{\mathbf{F}}_{0}\right) \cdot \hat{\mathbf{F}}_{0}^{\prime \prime}$.

Now, from (7),

$\hat{\mathbf{F}}=\mathbf{F}+\mathbf{d} \otimes \mathbf{k}, \quad \hat{\mathbf{F}}^{\prime}=\mathbf{D}+\mathbf{g} \otimes \mathbf{k} \quad$ and $\quad \hat{\mathbf{F}}^{\prime \prime}=\mathbf{G}+\mathbf{h} \otimes \mathbf{k}$

with

$\mathbf{D}=\mathbf{F}^{\prime}, \quad \mathbf{G}=\mathbf{D}^{\prime}, \quad \mathbf{g}=\mathbf{d}^{\prime} \quad$ and $\quad \mathbf{h}=\mathbf{g}^{\prime}$.

Using these with (10) and the symmetry of crossed partials furnishes

$\mathbf{D}=(\nabla \hat{\mathbf{y}})^{\prime}=\nabla \mathbf{d} \quad$ and $\quad \mathbf{G}=(\nabla \mathbf{d})^{\prime}=\nabla \mathbf{g}$.

We thus obtain

$\mathbf{F}_{0}=\nabla\left(\hat{\mathbf{y}}_{0}\right), \quad \mathbf{D}_{0}=\nabla\left(\mathbf{d}_{0}\right), \quad \mathbf{G}_{0}=\nabla\left(\mathbf{g}_{0}\right) \quad$ and $\quad \mathbf{h}_{0}=\mathbf{g}_{0}^{\prime}$,

in which it is emphasized that $\hat{\mathbf{y}}_{0}, \mathbf{d}_{0}, \mathbf{g}_{0}$ and $\mathbf{h}_{0}$ are independent functions of $\mathbf{u}$.
Henceforth we suppress the subscript ()$_{0}$ and write the strain energy as

$\mathscr{E}=\int_{\kappa} U(\tilde{\mathbf{F}}(\mathbf{x})) \mathrm{d} V=E+O\left(\varepsilon^{5}\right)$,

where

$E=\int_{\Omega} W(\mathbf{d}, \mathbf{g}, \mathbf{h}, \mathbf{F}, \mathbf{D}, \mathbf{G}) \mathrm{d} A$

in which

$$
\begin{aligned}
W= & \varepsilon U(\mathbf{F}+\mathbf{d} \otimes \mathbf{k})+\frac{1}{24} \varepsilon^{3}\{\mathbf{P}(\mathbf{F}+\mathbf{d} \otimes \mathbf{k}) \cdot(\mathbf{G}+\mathbf{h} \otimes \mathbf{k}) \\
& +\mathscr{M}(\mathbf{F}+\mathbf{d} \otimes \mathbf{k})[\mathbf{D}+\mathbf{g} \otimes \mathbf{k}] \cdot(\mathbf{D}+\mathbf{g} \otimes \mathbf{k})\}
\end{aligned}
$$

is the strain energy through order $O\left(\varepsilon^{4}\right)$ per unit area of $\Omega$, $\mathbf{r}(\mathbf{u})\left(=\hat{\mathbf{y}}_{0}\right)$ is the map from the plate midsurface $\Omega$ to its deformed image $\omega$, and $\mathbf{d}(\mathbf{u}), \mathbf{g}(\mathbf{u}), \mathbf{h}(\mathbf{u})$ are director fields defined on $\Omega$. These are the coefficient vectors in the expansion

$\hat{\mathbf{y}}(\mathbf{u}, \varsigma)=\mathbf{r}(\mathbf{u})+\varsigma \mathbf{d}(\mathbf{u})+\frac{1}{2} \varsigma^{2} \mathbf{g}(\mathbf{u})+\frac{1}{6} \varsigma^{3} \mathbf{h}(\mathbf{u})+O\left(\varsigma^{4}\right)$,

and $\mathbf{F}=\nabla \mathbf{r}$ maps $\Omega^{\prime}$ to the tangent plane $T_{\omega(\mathbf{u})}$ of the deformed midsurface $\omega$ at the point $\mathbf{r}(\mathbf{u})$.

\section{Membrane theory and inextensional bending theory}

Membrane theory and inextensional bending theory have recently been the focus of intensive research on rigorous smallthickness limits of three-dimensional elasticity. Two main lines of inquiry, one based on the method of asymptotic expansions (see [6-8]) and the other on the method of Gamma convergence (see $[2-5,12]$ ) have succeeded in putting both models on firm mathematical foundations. Before developing the full theory for combined stretching and bending, we show in the present section that the membrane and inextensional bending energies are subsumed under the expression (20).

\subsection{Membrane theory}

Thus, membrane theory emerges in the limit

$E_{m}=\lim _{\varepsilon \rightarrow 0} \varepsilon^{-1} \mathscr{E}=\int_{\Omega} M(\mathbf{F}, \mathbf{d}) \mathrm{d} A$,

where

$M(\mathbf{F}, \mathbf{d})=U(\mathbf{F}+\mathbf{d} \otimes \mathbf{k})$

is the membrane strain-energy function. To find the gradients of this function we consider a one-parameter family of deformations parametrized by a real variable $u$ and use superposed dots to denote the associated derivatives, evaluated at $u=0$, say. Thus, from (1) and (8),

$M_{\mathbf{F}} \cdot \dot{\mathbf{F}}+M_{\mathbf{d}} \cdot \dot{\mathbf{d}}=\mathbf{P}(\mathbf{F}+\mathbf{d} \otimes \mathbf{k}) \mathbf{1} \cdot \dot{\mathbf{F}}$

$$
+\mathbf{P}(\mathbf{F}+\mathbf{d} \otimes \mathbf{k}) \mathbf{k} \cdot \dot{\mathbf{d}} \text {. }
$$

Then

$\mathbf{P}(\mathbf{F}+\mathbf{d} \otimes \mathbf{k}) \mathbf{1}=M_{\mathbf{F}} \quad$ and $\quad \mathbf{P}(\mathbf{F}+\mathbf{d} \otimes \mathbf{k}) \mathbf{k}=M_{\mathbf{d}}$ 
The Euler equations are

$\operatorname{div} M_{\mathbf{F}}=\mathbf{0}$ and $M_{\mathbf{d}}=\mathbf{0}$ in $\Omega$,

wherein div is the two-dimensional divergence with respect to $\mathbf{u} \in \Omega$, while typical boundary conditions entail the specification of $\mathbf{r}$ and $\left(M_{\mathbf{F}}\right) \boldsymbol{v}$ on complementary parts of $\partial \Omega$, where $\boldsymbol{v}$ is the exterior unit normal to the latter in the sense of Green's theorem. From $(26)_{2}$ and $(27)_{2}$ it follows that no tractions are transmitted across the midsurface, i.e.

$\mathbf{P}(\mathbf{F}+\mathbf{d} \otimes \mathbf{k}) \mathbf{k}=\mathbf{0}$.

This in turn furnishes a relationship between $\mathbf{d}$ and $\mathbf{F}$ which is uniformly valid over $\Omega$. The derivative of the left-hand side with respect to $\mathbf{d}$ is the acoustic tensor $\mathbf{A}$, with components given by

$A_{i j}=\mathscr{M}_{i 3 j 3}=\mathscr{M}_{i A j B} k_{A} k_{B}$,

in which $\mathscr{M}$ is evaluated at $\mathbf{F}+\mathbf{d} \otimes \mathbf{k}$. Our assumption that strong ellipticity holds at equilibrium states implies that this is positive definite at equilibrium values of the argument. The implicit function theorem then ensures that (28) can be solved uniquely for the associated values of $\mathbf{d}$ in the form $[6,7,14,15]$

$\mathbf{d}=\overline{\mathbf{d}}(\mathbf{F})$.

For example, it is known that if the three-dimensional strainenergy function possesses reflection symmetry with respect to the midsurface $\Omega$, then $\overline{\mathbf{d}}=\lambda \mathbf{n}$, where $\lambda$ is a scalar-valued function of $\mathbf{F}$ and $\mathbf{n}$ is a unit normal to the tangent plane $T_{\omega(\mathbf{u})}$ to the deformed midsurface [14,15]. Substitution into (26) 1 and $(27)_{1}$ furnishes a problem for the determination of the midsurface position field $\mathbf{r}(\mathbf{u})$.

It transpires that the function $M(\cdot, \overline{\mathbf{d}}(\cdot))$ fails to satisfy the relevant (two-dimensional) Legendre-Hadamard inequality (semi-strict strong ellipticity) and thus fails to be quasiconvex, even when $U$ is strongly elliptic in the three-dimensional sense. This is due to the presence of compressive stresses in the stress-deformation relation $(26)_{1}$, whereas compressive stresses are ruled out by the Legendre-Hadamard condition [18]. For this reason equilibrium boundary-value problems for a membrane theory based on (23) will generally fail to possess energy-minimizing solutions. In such circumstances well-posedness may be restored via relaxation, in which the function $M(\cdot, \overline{\mathbf{d}}(\cdot))$ is replaced by its quasiconvexification [17]; i.e., the largest quasiconvex function nowhere exceeding the original function on its domain. One then proceeds as usual with the Euler equations based on the relaxed problem [18,19]. Precisely the same result is obtained by the method of Gamma convergence [12].

\subsection{Inextensional bending theory}

To recover inextensional bending theory we assume that $U(\mathbf{I})=0$ and $\mathbf{P}(\mathbf{I})=\mathbf{0}$. For frame-invariant strain-energy functions we then have $U(\mathbf{Q})=0$ and $\mathbf{P}(\mathbf{Q})=\mathbf{0}$ for all rotations $\mathbf{Q}$. These follow from the general rules $U(\mathbf{Q} \tilde{\mathbf{F}})=U(\tilde{\mathbf{F}})$ and $\mathbf{P}(\mathbf{Q} \tilde{\mathbf{F}})=\mathbf{Q P}(\tilde{\mathbf{F}})$, respectively [20]. Inextensional bending is associated with pure rotation of material on the midsurface. In the treatment given in [2-4], $U(\tilde{\mathbf{F}})$ is required to be bounded below by the squared distance between $\tilde{\mathbf{F}}$ and the group of rotations, so that it is positive definite as a function of strain. Thus inextensional bending is energetically optimal for points on the midsurface whenever it is allowed by boundary conditions. In this case $\left.\tilde{\mathbf{F}}\right|_{\varsigma=0}$ reduces to the rotation factor in its polar decomposition; we write $\hat{\mathbf{F}}(\mathbf{u}, 0)=\mathbf{R}(\mathbf{u})$. From $(15)_{1}$ we then have

$\mathbf{F}+\mathbf{d} \otimes \mathbf{k}=\mathbf{R}$

so that

$\mathbf{F}=\mathbf{R} \mathbf{1}$ and $\mathbf{d}=\mathbf{R} \mathbf{k}=\mathbf{n}$,

where $\mathbf{n}(\mathbf{u})$ is a unit normal to $\left.T_{\omega(\mathbf{u}}\right)$, this following from

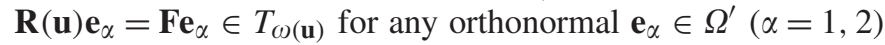
and the fact that $\mathbf{R}$ preserves the orthonormality and handedness of $\left\{\mathbf{e}_{\alpha}, \mathbf{k}\right\}$. The first of (32) gives $\mathbf{F}^{\mathrm{t}} \mathbf{F}=\mathbf{1}$, so that $\mathbf{r}(\mathbf{u})$ is an isometry, while the second implies that transverse fibers located on $\Omega$ suffer no extension or contraction and no shear relative to fibers lying in $\Omega^{\prime}$. The energy associated with inextensional bending is thus given by

$E_{\mathrm{b}}=\lim _{\varepsilon \rightarrow 0} \varepsilon^{-3} \mathscr{E}=\varepsilon^{-3} E=\int_{\Omega} B(\nabla \mathbf{n} ; \mathbf{R}, \mathbf{g}) \mathrm{d} A$,

where

$B(\nabla \mathbf{n} ; \mathbf{R}, \mathbf{g})=\frac{1}{24} \mathscr{M}(\mathbf{R})[\nabla \mathbf{n}+\mathbf{g} \otimes \mathbf{k}] \cdot(\nabla \mathbf{n}+\mathbf{g} \otimes \mathbf{k})$.

The Euler equation associated with variations in $\mathbf{g}$ is given simply by $B_{\mathbf{g}}=\mathbf{0}$, which is equivalent to

$\{\mathscr{M}(\mathbf{R})[\nabla \mathbf{n}+\mathbf{g} \otimes \mathbf{k}]\} \mathbf{k}=\mathbf{0}$.

If strong ellipticity is satisfied in the three-dimensional sense, then the solution

$\mathbf{g}=\overline{\mathbf{g}}(\nabla \mathbf{n}, \mathbf{R})$

is unique and minimizes $B(\nabla \mathbf{n} ; \mathbf{R}, \cdot)$. To see this we write (35) in the form

$\mathbf{A}(\mathbf{R}) \mathbf{g}=-\{\mathscr{M}(\mathbf{R})[\nabla \mathbf{n}]\} \mathbf{k}$,

where $\mathbf{A}(\mathbf{R})$ the acoustic tensor defined by (29) with $\mathscr{M}$ evaluated at $\mathbf{R}$. Our hypotheses imply that this is positive definite; (36) then follows immediately from (37). We note that the solution $\overline{\mathbf{g}}$ delivered by (37) does not conflict with the kinematic requirement that the determinant of the deformation gradient be positive. In the present case this requirement may be cast in the form

$1+\varsigma \mathbf{R} \cdot(\nabla \mathbf{n}+\mathbf{g} \otimes \mathbf{k})+o(\varsigma)>0 ; \quad \varsigma \in(-\varepsilon / 2, \varepsilon / 2)$,

which is assured for all $\mathbf{g}$ if $\varepsilon$ is sufficiently small.

To prove that (36) is optimal we define $R(\cdot) / 12:=$ $B(\nabla \mathbf{n} ; \mathbf{R}, \cdot)$. If $\mathbf{g}(u)$ is a regular curve in $E^{3}$ with no points of self-intersection, then the derivatives of $\sigma(u)=R(\mathbf{g}(u))$ with respect to $u$ are

$\dot{\sigma}=\dot{\mathbf{g}} \cdot\{\mathscr{M}(\mathbf{R})[\nabla \mathbf{n}+\mathbf{g} \otimes \mathbf{k}]\} \mathbf{k}=\dot{\mathbf{g}} \cdot R_{\mathbf{g}}$ 
and

$$
\begin{aligned}
\ddot{\sigma} & =\ddot{\mathbf{g}} \cdot\{\mathscr{M}(\mathbf{R})[\nabla \mathbf{n}+\mathbf{g} \otimes \mathbf{k}]\} \mathbf{k}+\dot{\mathbf{g}} \otimes \mathbf{k} \cdot \mathscr{M}(\mathbf{R})[\dot{\mathbf{g}} \otimes \mathbf{k}] \\
& =\ddot{\mathbf{g}} \cdot R_{\mathbf{g}}+\dot{\mathbf{g}} \cdot\left(R_{\mathbf{g g}}\right) \dot{\mathbf{g}},
\end{aligned}
$$

where we have used the major symmetry of $\mathscr{M}$. Thus,

$R_{\mathbf{g}}=\{\mathscr{M}(\mathbf{R})[\nabla \mathbf{n}+\mathbf{g} \otimes \mathbf{k}]\} \mathbf{k}$

vanishes by (35), whereas

$R_{\mathrm{gg}}=\mathbf{A}(\mathbf{R})$.

Strong ellipticity ensures that this is positive definite and thus that $\ddot{\sigma}>0$ on straight-line paths defined by $\mathbf{g}(u)=u \mathbf{g}_{2}+$ $(1-u) \mathbf{g}_{1}$ with $\mathbf{g}_{1}, \mathbf{g}_{2}$ fixed and $0 \leqslant u \leqslant 1$. These paths belong to the convex set $E^{3}$, the domain of $R(\cdot)$. Integrating with respect to $u$ yields $\dot{\sigma}(u)>\dot{\sigma}(0)$ and $\sigma(1)-\sigma(0)>\dot{\sigma}(0)$, proving that $R(\mathbf{g})$ is strictly convex, i.e.

$R\left(\mathbf{g}_{2}\right)-R\left(\mathbf{g}_{1}\right)>R_{\mathbf{g}}\left(\mathbf{g}_{1}\right) \cdot\left(\mathbf{g}_{2}-\mathbf{g}_{1}\right)$

for all unequal pairs $\mathbf{g}_{1}, \mathbf{g}_{2}$. It follows immediately that $R$ is minimized absolutely at a stationary point and thus that the solution $\overline{\mathbf{g}}$ to (35) is energetically optimal. The uniqueness of $\overline{\mathbf{g}}$ may be proved from (43) and is immediately apparent from (37).

The expression for $B$ may be simplified by exploiting the frame invariance of the constitutive equations [20]. To see this we again consider a one-parameter family of deformations. Thus, for any fixed rotation $\mathbf{Q}$ we have

$\mathbf{P}(\mathbf{Q H}) \cdot \mathbf{Q} \dot{\mathbf{H}}=\dot{U}(\mathbf{Q H})=\dot{U}(\mathbf{H})=\mathbf{P}(\mathbf{H}) \cdot \dot{\mathbf{H}}$.

Differentiating the outer equality at fixed $\dot{\mathbf{H}}$ yields

$\mathscr{M}(\mathbf{Q H})[\mathbf{Q} \dot{\mathbf{H}}] \cdot \mathbf{Q} \dot{\mathbf{H}}=\mathscr{M}(\mathbf{H})[\dot{\mathbf{H}}] \cdot \dot{\mathbf{H}}$.

Applying this with $\mathbf{Q}=\mathbf{R}, \mathbf{H}=\mathbf{I}$ and $\dot{\mathbf{H}}=\mathbf{S}$, where

$\mathbf{S}=\mathbf{R}^{\mathrm{t}}[\nabla \mathbf{n}+\overline{\mathbf{g}} \otimes \mathbf{k}]$,

then furnishes

$\mathscr{M}(\mathbf{R})[\nabla \mathbf{n}+\overline{\mathbf{g}} \otimes \mathbf{k}] \cdot(\nabla \mathbf{n}+\overline{\mathbf{g}} \otimes \mathbf{k})=\mathscr{M}(\mathbf{I})[\mathbf{S}] \cdot \mathbf{S}$.

To interpret the bending strain $\mathbf{S}$ we combine $\mathrm{d} \mathbf{n}=(\nabla \mathbf{n}) \mathrm{d} \mathbf{u}$ and $\mathrm{d} \mathbf{r}=(\nabla \mathbf{r}) \mathrm{d} \mathbf{u}$ with $\mathrm{d} \mathbf{n}=-\mathbf{b} \mathrm{d} \mathbf{r}$, where $\mathbf{b}$ is the (symmetric) curvature tensor on $T_{\omega(\mathbf{u})}$ [8], to derive $\nabla \mathbf{n}=-\mathbf{b F}$. This maps $\Omega^{\prime}$ to $T_{\omega(\mathbf{u})}$ because $\mathbf{n} \cdot \mathrm{d} \mathbf{n}=0$. Further, from the properties of $\mathbf{R}$ it follows that $\mathbf{R}^{\mathrm{t}}$ maps $T_{\omega(\mathbf{u})}$ to $\Omega^{\prime}$ and thus that

$\mathbf{R}^{\mathrm{t}} \nabla \mathbf{n}=-\mathbf{F}^{\mathrm{t}} \mathbf{b} \mathbf{F}$.

With this result the bending energy

$E_{\mathrm{b}}=\frac{1}{24} \int_{\Omega} \mathscr{M}(\mathbf{I})[\mathbf{S}] \cdot \mathbf{S} \mathrm{d} A$

is seen to be identical to that obtained in [2,3] by the method of Gamma convergence. For isotropic materials the solution obtained in [2] for $\mathbf{g}=\overline{\mathbf{g}}$ exists if $\mathscr{M}$ (I) satisfies the strong-ellipticity condition imposed here. Specifically, the solution cited requires that $\lambda+2 \mu \neq 0$, where $\lambda$ and $\mu$ are the classical Lamé moduli for isotropic materials, this being guaranteed by strong ellipticity. The Euler equations and boundary conditions associated with $E_{\mathrm{b}}$ are developed in [6,7].

Gauss' Theorema Egregium requires that the Gaussian curvature, det $\mathbf{b}$, be everywhere equal to its value on the reference configuration and hence that it vanish identically. The bending energy, being valid for isometries only, fails of course to furnish a model for combined stretching and flexure of the midsurface. It does model stiff sheets, such as paper, which tear or crumple rather than incur the large energetic penalty accompanying midsurface strain.

We note that the foregoing expressions for the membrane and bending energies incorporate partial information about equilibria in the form of the constraints $\mathbf{d}=\overline{\mathbf{d}}$ and $\mathbf{g}=\overline{\mathbf{g}}$, respectively. Moreover, these constraints are energetically optimal in their respective contexts. Their adoption therefore does not impede the attainment of the optimal overall energy. This fact justifies typical approaches to the variational theory for equilibrium problems in which such constraints are imposed on all geometrically possible configurations [2,3,13-15,18], as in the foregoing, followed by minimization of the resulting expression for the energy with respect to the remaining free variables. We show in Section 4 that similar restrictions emerge as Euler equations in the case of combined bending and stretching. However, they do not in general furnish optimal values of the local energy in that context. In particular, the method used is not specific to energy minimizers and may thus be extended to a dynamical setting in which the notion of energy minimization is irrelevant. It also accommodates equilibrium states that need not be energetically optimal.

\section{Combined bending and stretching}

In the general case the appropriate functional is

$E[\mathbf{r}, \mathbf{d}, \mathbf{g}, \mathbf{h}]=\int_{\Omega} W(\mathbf{d}, \mathbf{g}, \mathbf{h}, \mathbf{F}, \mathbf{D}, \mathbf{G}) \mathrm{d} A$,

where $W$ is given by (21). We have in mind the conventional idea that equilibria should be energy minimizers. This identification is possible only if the energy has a lower bound. To explore the implications, consider the choice

$\mathbf{h}=-\alpha \mathbf{P}(\mathbf{F}+\mathbf{d} \otimes \mathbf{k}) \mathbf{k}$

for some continuous function $\alpha(\mathbf{u})>0$. From the expression (21) for $W$, the associated term in the energy is proportional to $-\alpha|\mathbf{P}(\mathbf{F}+\mathbf{d} \otimes \mathbf{k}) \mathbf{k}|^{2}$, which is arbitrarily large and negative in the limit $\alpha \rightarrow \infty$ unless Pk vanishes identically. For pure traction problems this choice of $\mathbf{h}$ is admissible with $\alpha(\mathbf{u})$ constant. For mixed traction/placement (51) is admissible if $\alpha$ tapers off to zero on the boundary, and the argument then requires that $\max _{\mathbf{u} \in \Omega} \alpha(\mathbf{u}) \rightarrow \infty$. For the energy to have a lower bound it 
$\mathbf{P}(\mathbf{F}+\mathbf{d} \otimes \mathbf{k}) \mathbf{k}=\mathbf{0}$,

whether or not the deformation is in equilibrium. If this restriction is adopted as a constraint on all deformations, then for reasons discussed in the previous section no generality is lost if the objective is to minimize the energy absolutely.

We use superposed dots to denote variational derivatives. These are derivatives, evaluated at an equilibrium state, with respect to a parameter pertaining to a one-parameter family of configurations. Equilibria render the energy stationary and thus satisfy $\dot{E}=0$; i.e.,

$$
\begin{aligned}
0= & \int_{\Omega}\left[\dot{\mathbf{d}} \cdot\left(W_{\mathbf{d}}-\operatorname{div} W_{\mathbf{D}}\right)+\dot{\mathbf{g}} \cdot\left(W_{\mathbf{g}}-\operatorname{div} W_{\mathbf{G}}\right)\right. \\
& \left.+\dot{\mathbf{h}} \cdot W_{\mathbf{h}}-\dot{\mathbf{r}} \cdot \operatorname{div} W_{\mathbf{F}}\right] \mathrm{d} A \\
& +\int_{\partial \Omega}\left[\dot{\mathbf{r}} \cdot\left(W_{\mathbf{F}}\right) \boldsymbol{v}+\dot{\mathbf{d}} \cdot\left(W_{\mathbf{D}}\right) \boldsymbol{v}+\dot{\mathbf{g}} \cdot\left(W_{\mathbf{G}}\right) \boldsymbol{v}\right] \mathrm{d} S,
\end{aligned}
$$

and the Euler equations are

$\operatorname{div} W_{\mathbf{F}}=\mathbf{0}, \quad W_{\mathbf{d}}=\operatorname{div} W_{\mathbf{D}}, \quad W_{\mathbf{g}}=\operatorname{div} W_{\mathbf{G}} \quad$ and $\quad W_{\mathbf{h}}=\mathbf{0}$.

From (21), the last of these is seen to be equivalent to (52). Accordingly, the necessary (52) for the existence of a lower bound on the energy is also a stationarity condition. This has important implications for dynamical problems. Since dynamical states are not energy minimizers, there is no reason to require the existence of a lower bound on the total strain energy, and thus no reason to impose (52) at the outset. The framework of the present section would thus seem to lend itself more naturally to the study of dynamical problems. This will be pursued in detail elsewhere.

To interpret the terms in (54) we use the chain rule and (21) to obtain

$$
\begin{aligned}
& W_{\mathbf{d}} \cdot \dot{\mathbf{d}}+W_{\mathbf{D}} \cdot \dot{\mathbf{D}}+W_{\mathbf{g}} \cdot \dot{\mathbf{g}}+W_{\mathbf{G}} \cdot \dot{\mathbf{G}}+W_{\mathbf{F}} \cdot \dot{\mathbf{F}}+W_{\mathbf{h}} \cdot \dot{\mathbf{h}} \\
&= \dot{W} \\
&= \varepsilon \mathbf{P} \cdot(\dot{\mathbf{F}}+\dot{\mathbf{d}} \otimes \mathbf{k})+\frac{\varepsilon^{3}}{24}\{\mathscr{M}[\mathbf{G}+\mathbf{h} \otimes \mathbf{k}] \cdot(\dot{\mathbf{F}}+\dot{\mathbf{d}} \otimes \mathbf{k}) \\
&+\mathscr{A}[\mathbf{D}+\mathbf{g} \otimes \mathbf{k}][\mathbf{D}+\mathbf{g} \otimes \mathbf{k}] \cdot(\dot{\mathbf{F}}+\dot{\mathbf{d}} \otimes \mathbf{k}) \\
&+\mathbf{P} \cdot(\dot{\mathbf{G}}+\dot{\mathbf{h}} \otimes \mathbf{k})+2 \mathscr{M}[\mathbf{D}+\mathbf{g} \otimes \mathbf{k}] \cdot(\dot{\mathbf{D}}+\dot{\mathbf{g}} \otimes \mathbf{k})\},
\end{aligned}
$$

where the stress and the first- and second-order moduli are evaluated at $\mathbf{F}+\mathbf{d} \otimes \mathbf{k}$. We have made use of certain symmetries in $\mathscr{M}$ and $\mathscr{A}$ that follow from (3) and (4). The second-order moduli yield a non-standard term arising from the variation of $\mathscr{M}(\mathbf{F}+\mathbf{d} \otimes \mathbf{k})$ in the expression (21) for the strain energy. This has no counterpart in inextensional bending theory as the

$$
\begin{aligned}
W_{\mathbf{h}}= & \frac{\varepsilon^{3}}{24} \mathbf{P k}, \quad W_{\mathbf{g}}=\frac{\varepsilon^{3}}{24} 2\{\mathscr{M}[\mathbf{D}+\mathbf{g} \otimes \mathbf{k}]\} \mathbf{k}, \\
W_{\mathbf{G}}= & \frac{\varepsilon^{3}}{24} \mathbf{P 1}, \quad W_{\mathbf{D}}=\frac{\varepsilon^{3}}{24} 2\{\mathscr{M}[\mathbf{D}+\mathbf{g} \otimes \mathbf{k}]\} \mathbf{1}, \\
W_{\mathbf{d}}= & \varepsilon \mathbf{P k}+\frac{\varepsilon^{3}}{24}\{\mathscr{M}[\mathbf{G}+\mathbf{h} \otimes \mathbf{k}]\} \mathbf{k} \\
& +\frac{\varepsilon^{3}}{24}\{\mathscr{A}[\mathbf{D}+\mathbf{g} \otimes \mathbf{k}][\mathbf{D}+\mathbf{g} \otimes \mathbf{k}]\} \mathbf{k}, \\
W_{\mathbf{F}}= & \varepsilon \mathbf{P} \mathbf{1}+\frac{\varepsilon^{3}}{24}\{\mathscr{M}[\mathbf{G}+\mathbf{h} \otimes \mathbf{k}]\} \mathbf{1} \\
& +\frac{\varepsilon^{3}}{24}\{\mathscr{A}[\mathbf{D}+\mathbf{g} \otimes \mathbf{k}][\mathbf{D}+\mathbf{g} \otimes \mathbf{k}]\} \mathbf{1} .
\end{aligned}
$$

The Euler equations then furnish the system

$$
\mathbf{P k}=\mathbf{0},
$$

$$
\begin{aligned}
\{\mathscr{M}[\mathbf{D}+\mathbf{g} \otimes \mathbf{k}]\} \mathbf{k}= & \frac{1}{2} \operatorname{div}(\mathbf{P} \mathbf{1}) \\
\{\mathscr{M}[\mathbf{G}+\mathbf{h} \otimes \mathbf{k}]\} \mathbf{k}= & 2 \operatorname{div}(\{\mathscr{M}[\mathbf{D}+\mathbf{g} \otimes \mathbf{k}]\} \mathbf{1}) \\
& -\{\mathscr{A}[\mathbf{D}+\mathbf{g} \otimes \mathbf{k}][\mathbf{D}+\mathbf{g} \otimes \mathbf{k}]\} \mathbf{k}
\end{aligned}
$$

and

$$
\begin{aligned}
\mathbf{0}= & \frac{\varepsilon^{2}}{24} \operatorname{div}\{\mathscr{M}[\mathbf{G}+\mathbf{h} \otimes \mathbf{k}] \mathbf{1}+\mathscr{A}[\mathbf{D}+\mathbf{g} \otimes \mathbf{k}][\mathbf{D}+\mathbf{g} \otimes \mathbf{k}] \mathbf{1}\} \\
& +\operatorname{div}(\mathbf{P} \mathbf{1}) .
\end{aligned}
$$

The component forms are

$$
\begin{gathered}
P_{i 3}=0, \quad A_{i j} g_{j}=\frac{1}{2} P_{i \alpha, \alpha}-\mathscr{M}_{i 3 j \beta} d_{j, \beta}, \\
A_{i j} h_{j}=2\left(\mathscr{M}_{i \alpha j \beta} d_{j, \beta}+\mathscr{M}_{i \alpha j 3} g_{j}\right)_{, \alpha}-\mathscr{M}_{i 3 j \beta} g_{j, \beta} \\
-\mathscr{A}_{i 3 j \beta k \gamma} d_{j, \beta} d_{k, \gamma}-\mathscr{A}_{i 3 j 3 k \gamma} g_{j} d_{k, \gamma} \\
-\mathscr{A}_{i 3 j \beta k 3} d_{j, \beta} g_{k}-\mathscr{A}_{i 3 j k 3} g_{j} g_{k},
\end{gathered}
$$

and

$\frac{\varepsilon^{2}}{24} T_{i \alpha, \alpha}+P_{i \alpha, \alpha}=0$

where

$$
\begin{aligned}
T_{i \alpha}= & \mathscr{M}_{i \alpha j \beta} g_{j, \beta}+\mathscr{M}_{i \alpha j 3} h_{j}+\mathscr{A}_{i \alpha j \beta k \gamma} d_{j, \beta} d_{k, \gamma} \\
& +\mathscr{A}_{i \alpha j 3 k \gamma} g_{j} d_{k, \gamma}+\mathscr{A}_{i \alpha j \beta k 3} d_{j, \beta} g_{k}+\mathscr{A}_{i \alpha j 3 k 3} g_{j} g_{k}
\end{aligned}
$$

and

$P_{i \alpha, \alpha}=\mathscr{M}_{i \alpha j \beta} r_{j, \beta \alpha}+\mathscr{M}_{i \alpha j 3} d_{j, \alpha}$,

where Greek indices take values in $\{1,2\}$.

Under the strong-ellipticity hypothesis equations (61) 1,2 and (62) furnish a decoupled algebraic system which may be used to eliminate $\mathbf{d}, \mathbf{g}$ and $\mathbf{h}$ in favor of $\mathbf{r}$ and its derivatives. These may be used in (63) to obtain a set of three equations for (the components of) $\mathbf{r}$. The resulting system is of the fourth order in $\mathbf{r}(\mathbf{u})$.

The Euler equations involve the spatial derivatives $d_{i, \alpha}$. These may be expressed in terms of the derivatives of the midsurface deformation function $\mathbf{r}(\mathbf{u})$. To this end we observe that 
the function $\overline{\mathbf{d}}(\mathbf{F})$ satisfies (57) identically in $\mathbf{F}$. Differentiation of this identity yields

$\mathscr{M}_{i 3 j \beta}+A_{i k} K_{k j \beta}=0$,

where

$K_{k j \beta}=\partial \bar{d}_{k} / \partial F_{j \beta}$.

Accordingly [14],

$K_{k j \beta}=-A_{k i}^{-1} \mathscr{M}_{i 3 j \beta}$,

where the right-hand side is evaluated at $\mathbf{F}+\overline{\mathbf{d}}(\mathbf{F}) \otimes \mathbf{k}$, yielding

$d_{i, \alpha}=K_{i j \beta} r_{j, \beta \alpha}$.

\section{Edge conditions}

Boundary conditions for this model may be deduced from (53). For example, if the three-dimensional deformation is assigned on the cylindrical surface $\partial \Omega_{\mathrm{c}} \times(-\varepsilon / 2, \varepsilon / 2)$, then its dependence on the through-thickness coordinate $\varsigma$ is prescribed. Differentiating this function with respect to $\varsigma$ and evaluating it at $\varsigma=0$ leads to the conclusion that the restrictions to $\partial \Omega_{\mathrm{c}}$ of $\mathbf{r}, \mathbf{d}, \mathbf{g}$ and $\mathbf{h}$ are also prescribed, and thus that the variations $\dot{\mathbf{r}}, \dot{\mathbf{d}}, \dot{\mathbf{g}}$ and $\dot{\mathbf{h}}$ vanish on $\partial \Omega_{\mathrm{c}}$. We refer to this as a clamped boundary. It is clear that this condition is somewhat non-standard in the sense that the boundary values of $\mathbf{d}, \mathbf{g}$ and $\mathbf{h}$ cannot be fixed arbitrarily. For, such data cannot be expected to agree with the continuous extensions to $\partial \Omega_{\mathrm{c}}$ of the equilibrium fields $\mathbf{d}, \mathbf{g}$ and $\mathbf{h}$ delivered by the algebraic formulae $(61)_{1,2}$ and (62), respectively. To avoid the non-existence of solutions implied by this circumstance, we stipulate that the assigned boundary data for $\mathbf{d}, \mathbf{g}$ and $\mathbf{h}$ be fixed at the values furnished by the continuous extensions to $\partial \Omega_{\mathrm{c}}$ of the respective equilibrium fields, while the boundary values of $\mathbf{r}$ are specified as genuine data a priori. In effect this procedure entails the simultaneous assignment of $\mathbf{r}$ and its normal derivative $\mathbf{r}_{v}$ on a clamped boundary. To see this we write

$\nabla \mathbf{r}=\mathbf{r}^{\prime} \otimes \tau+\mathbf{r}_{v} \otimes v$,

where $\tau$ and $\boldsymbol{v}$ are the unit tangent and normal to $\partial \Omega_{\mathrm{c}}$, arranged such that $\{\boldsymbol{v}, \tau, \mathbf{k}\}$ has positive orientation, and $\mathbf{r}^{\prime}$ is the arclength derivative of $\mathbf{r}$ on $\partial \Omega_{\mathrm{c}}$. The arclength derivative $\mathbf{r}^{\prime}$ is fixed by the values of $\mathbf{r}$ on $\partial \Omega_{\mathrm{c}}$. The boundary values of the equilibrium field $\mathbf{d}=\overline{\mathbf{d}}(\nabla \mathbf{r})$ are thus controlled by those of $\mathbf{r}$ and $\mathbf{r}_{v}$. Boundary data of this type also occur in the theory of Hilgers and Pipkin [13-15], in which a relation like $\mathbf{d}=\overline{\mathbf{d}}(\nabla \mathbf{r})$ is imposed on all configurations, not merely those that render the energy stationary. In our approach, we take the view that boundary values of $\mathbf{g}$ and $\mathbf{h}$ are delivered a posteriori by the solutions to the equilibrium problem. For the resulting model to represent actual position data on the boundary of a thin threedimensional body, it is thus necessary that the boundary effectively adjust to the problem at hand, in a way that does not conform to the usual intention when such data are imposed. This is a price to be paid for using a two-dimensional theory to represent three-dimensional behavior.
The deformation is unrestricted on a traction-free cylindrical surface $\partial \Omega_{t} \times(-\varepsilon / 2, \varepsilon / 2)$ of the thin body. Accordingly, the manner in which the deformation function varies with $\varsigma$ is similarly unrestricted. It follows from this that $\dot{\mathbf{r}}, \dot{\mathbf{d}}, \dot{\mathbf{g}}$ and $\dot{\mathbf{h}}$ are unrestricted on $\partial \Omega_{t}$ in principle. The associated natural boundary conditions may be read off from (53); these require that $\left(W_{\mathbf{F}}\right) \boldsymbol{v},\left(W_{\mathbf{D}}\right) \boldsymbol{v}$ and $\left(W_{\mathbf{G}}\right) \boldsymbol{v}$ vanish on $\partial \Omega_{t}$. However, this results in an overdetermined problem. For, we have seen that the final system for $\mathbf{r}(\mathbf{u})$ is of the fourth order. Compatible data entail the specification of two vector conditions on a boundary part of given type. This is exemplified by the data on a clamped part of the boundary. To avoid overdeterminacy, we relax the requirement that $\left(W_{\mathbf{G}}\right) \boldsymbol{v}$ vanish and require only that $\left(W_{\mathbf{F}}\right) \boldsymbol{v}$ and $\left(W_{\mathbf{D}}\right) \boldsymbol{v}$ vanish. This is consistent with the variational statement (53) provided that $\dot{\mathbf{g}}$ vanishes on the boundary. In effect, we require $\mathbf{g}$ to be fixed at the values furnished by the continuous extension to the boundary of the equilibrium values of $\mathbf{g}$ in the interior, as before.

If dead loads are assigned on $\partial \Omega_{t} \times(-\varepsilon / 2, \varepsilon / 2)$, then the potential energy of the three-dimensional body is $\mathscr{E}-\mathscr{L}$, where $\mathscr{E}$ is the total strain energy defined by (19), and

$\mathscr{L}=\int_{\partial \Omega_{t}}\left(\int_{-\varepsilon / 2}^{\varepsilon / 2} \mathbf{p} \cdot \chi \mathrm{d} \varsigma\right) \mathrm{d} \mathbf{S}$

is the load potential in which $\mathbf{p}(\mathbf{x})=\hat{\mathbf{p}}(\mathbf{u}, \varsigma)$ is the assigned Piola traction. Using a formula like (13), it is straightforward to show that

$\mathscr{E}-\mathscr{L}=E-L+O\left(\varepsilon^{4}\right)$,

in which $E$ is defined by (20) and

$L=\int_{\partial \Omega_{t}} \chi(\mathbf{r}, \mathbf{d}, \mathbf{g}) \mathrm{d} \mathbf{S}$,

with

$\chi(\mathbf{r}, \mathbf{d}, \mathbf{g})=\left(\varepsilon \hat{\mathbf{p}}_{0}+\frac{\varepsilon^{3}}{24} \hat{\mathbf{p}}_{0}^{\prime \prime}\right) \cdot \mathbf{r}+\frac{\varepsilon^{3}}{24} 2 \hat{\mathbf{p}}_{0}^{\prime} \cdot \mathbf{d}+\frac{\varepsilon^{3}}{24} \hat{\mathbf{p}}_{0} \cdot \mathbf{g}$,

is the $O\left(\varepsilon^{3}\right)$ estimate of $\mathscr{L}$, where the primes now identify derivatives with respect to $\varsigma$ and the subscript ()$_{0}$ their values at $\varsigma=0$. To avoid overdeterminacy we impose $\dot{\mathbf{g}}=\mathbf{0}$ on the edge, as before, and arrive at the natural boundary conditions

$\left(W_{\mathbf{F}}\right) \boldsymbol{v}=\chi_{\mathbf{r}} \quad$ and $\quad\left(W_{\mathbf{D}}\right) \boldsymbol{v}=\chi_{\mathbf{d}} \quad$ on $\partial \Omega_{t}$,

where

$\chi_{\mathbf{r}}=\varepsilon \hat{\mathbf{p}}_{0}+\frac{\varepsilon^{3}}{24} \hat{\mathbf{p}}_{0}^{\prime \prime} \quad$ and $\quad \chi_{\mathbf{d}}=\frac{\varepsilon^{3}}{24} 2 \hat{\mathbf{p}}_{0}^{\prime}$.

Finally, we consider a part $\partial \Omega_{p}$ of the boundary to be pinned if the three-dimensional position on the surface $\partial \Omega_{p} \times(-\varepsilon / 2, \varepsilon / 2)$ is assigned only at $\varsigma=0$ while the remainder of the surface is traction free. Thus, $\mathbf{r}$ is assigned on $\partial \Omega_{p}$ whereas kinematic admissibility imposes no restriction on the midsurface value, $\mathbf{d}$, of the $\varsigma$-derivative of the three-dimensional deformation. Accordingly, $\dot{\mathbf{d}}$ is arbitrary and the natural boundary condition $\left(W_{\mathbf{D}}\right) \boldsymbol{v}=\mathbf{0}$ follows. 
Combinations of the foregoing are possible, and lead in a straightforward manner to relevant boundary data.

\section{Acknowledgments}

I thank T.J. Healey for helpful discussions about Gauss' Theorema Egregium in connection with inextensional bending theory. I also thank the referees for helpful comments leading to improvements in the manuscript.

\section{References}

[1] S.S. Antman, Nonlinear Problems of Elasticity, second ed., Springer, New York, 2005.

[2] G. Friesecke, R.D. James, S. Müller, A theorem on geometric rigidity and the derivation of nonlinear plate theory from three dimensional elasticity, Comm. Pure Appl. Math. 55 (2002) 1461-1506.

[3] G. Friesecke, S. Müller, R.D. James, Rigorous derivation of nonlinear plate theory and geometric rigidity, C. R. Acad. Sci. Paris, Ser. I 334 (2002) 173-178.

[4] G. Friesecke, R.D. James, S. Müller, A hierarchy of plate models derived from nonlinear elasticity by gamma-convergence, Arch. Ration. Mech. Anal. 180 (2006) 183-236.

[5] A. Braides, $\Gamma$-convergence for Beginners, Oxford University Press, Oxford, 2002.

[6] D.D. Fox, A. Raoult, J.C. Simo, A justification of nonlinear properly invariant plate theories, Arch. Rational. Mech. Anal. 124 (1993) 157-199.
[7] P.G. Ciarlet, Mathematical Elasticity, Vol. 3: Theory of Shells, NorthHolland, Amsterdam, 2000.

[8] P.G. Ciarlet, An introduction to differential geometry with applications to elasticity, J. Elasticity 78-79 (2005) 3-201.

[9] A. Libai, J.G. Simmonds, The Nonlinear Theory of Elastic Shells, second ed., Cambridge University Press, Cambridge, 1998.

[10] A. DiCarlo, P. Podio-Guidugli, W.O. Williams, Shells with thickness distension, Int. J. Solids Struct. 38 (2001) 1201-1225.

[11] P.M. Naghdi, Finite deformation of elastic rods and shells, in: D.E. Carlson, R.T. Shield (Eds.), Proceedings of the IUTAM Symposium on Finite Elasticity, Martinus Nijhoff, The Hague, 1981, pp. 47-101.

[12] H. Le Dret, A. Raoult, The membrane shell model in nonlinear elasticity: a variational asymptotic derivation, J. Nonlinear Sci. 6 (1996) 59-84.

[13] M.G. Hilgers, A.C. Pipkin, Elastic sheets with bending stiffness, Q. J. Mech. Appl. Math. 45 (1992) 57-75.

[14] M.G. Hilgers, A.C. Pipkin, Bending energy of highly elastic membranes, Q. Appl. Math. 50 (1992) 389-400.

[15] M.G. Hilgers, A.C. Pipkin, Bending energy of highly elastic membranes II, Quart. Appl. Math. 54 (1996) 307-316.

[16] R.W. Ogden, Non-Linear Elastic Deformations, Dover, New York, 1997.

[17] B. Dacorogna, Direct Methods in the Calculus of Variations, Springer, Berlin, 1989.

[18] A.C. Pipkin, The relaxed energy density for isotropic elastic membranes, IMA J. Appl. Math. 36 (1986) 85-99.

[19] D.J. Steigmann, Tension-field theory, Proc. R. Soc. Lond. A 429 (1990) $141-173$.

[20] A.I. Murdoch, Objectivity in classical continuum physics: a rationale for discarding the 'principle of invariance under superposed rigid body motions' in favour of purely objective considerations, Continuum Mech. Thermodyn. 15 (2003) 309-320. 